\title{
A LOG LOG IMPROVEMENT TO THE RIEMANN HYPOTHESIS FOR THE HAWKINS RANDOM SIEVE
}

\author{
By C. C. HEYDE
}

\section{CSIRO Division of Mathematics and Statistics, Canberra}

This paper is concerned with the Hawkins random sieve which is a probabilistic analogue of the sieve of Eratosthenes. Analogues of the prime number theorem, Mertens' theorem and the Riemann hypothesis have previously been established for the Hawkins sieve. In the present paper we give a more delicate analysis using iterated logarithm results for both martingales and tail sums of martingale differences to deduce a considerably improved $\log \log$ replacement for the Riemann hypothesis result.

1. Introduction. The random sieve introduced by Hawkins [2] is analogous to the sieve of Eratosthenes and produces a random sequence with asymptotic properties similar in many ways to the primes. It is constructed as follows: Let $A_{1}=\{2,3,4, \cdots\}$.

Stage 1. Put $X_{1}=\min A_{1}$. From the set $A_{1} \backslash\left\{X_{1}\right\}$ each number in turn is (independently of the others) deleted with probability $X_{1}^{-1}$ or not deleted with probability $1-X_{1}^{-1}$. The set of elements of $A_{1} \backslash\left\{X_{1}\right\}$ which remain is denoted by $A_{2}$.

Stage $n$. Put $X_{n}=\min A_{n}$. From the set $A_{n} \backslash\left\{X_{n}\right\}$ each number in turn is (independently of the others) deleted with probability $X_{n}{ }^{-1}$ or not deleted with probability $1-X_{n}{ }^{-1}$. The set of elements of $A_{n} \backslash\left\{X_{n}\right\}$ which remain is denoted by $A_{n+1}$.

Define

$$
Y_{n}=\prod_{1 \leqq k \leqq n}\left(1-X_{k}^{-1}\right)^{-1} .
$$

Wunderlich [8], [9] has obtained the results

$$
\lim _{n \rightarrow \infty}(n \log n)^{-1} X_{n}=1 \text { a.s. and } \lim _{n \rightarrow \infty}(\log n)^{-1} Y_{n}=1 \text { a.s. , }
$$

which are analogues of the prime number theorem and Mertens' theorem respectively, while Neudecker and Williams [6] have obtained an analogue of the Riemann hypothesis. Write

$$
\operatorname{li}(x)=\lim _{\delta \downarrow 0}\left(\int_{0}^{1-\delta}+\int_{1+\delta}^{x}\right) \frac{d z}{\log z} \sim \frac{x}{\log x} \quad \text { as } \quad x \rightarrow \infty,
$$

and recall that the "real" Riemann hypothesis about the zeros of the Riemann

Received April 14, 1977.

AMS 1970 subject classifications. Primary 60F 15, 10H30; Secondary 60G45, 60J05.

Key words and phrases. Random sieve, prime numbers, Riemann hypothesis, martingale iterated logarithm laws. 
zeta function is equivalent to

$$
\operatorname{li}\left(p_{n}\right)=n+O\left(n^{1+\varepsilon}\right)
$$

for any $\hat{\varepsilon}>0$ where $p_{n}$ denotes the $n$th prime. Neudecker and Williams showed that

$$
L \operatorname{li}\left(X_{n} L^{-1}\right)=n+O\left(n^{\frac{1}{2}+\varepsilon}\right) \text { a.s. , }
$$

for any $\varepsilon>0$ where $L$ denotes the (random) limit

$$
L=\lim _{n \rightarrow \infty} X_{n} \exp \left(-Y_{n}\right),
$$

which they show to exist and be nonzero a.s.

It is our object in this paper to sharpen this result to a $\log \log$ form as given in the following theorem.

TheOREM. For the Hawkins random sieve,

$$
\lim \sup _{n \rightarrow \infty}(2 \mathrm{n} \log \log n)^{-\frac{1}{2}}\left|L \operatorname{li}\left(X_{n} L^{-1}\right)-n\right| \leqq 3 \text { a.s. }
$$

The proof of this theorem rests heavily on the use of some recent results $([4],[5])$ on the law of the iterated logarithm for martingales and for tail sums of martingale differences. The result, of course, suggests a candidate for the bound in the "real" Riemann hypothesis. It should be remarked that a similar type of $\log \log$ error term also emerges for a structurally simpler diffusion analogue of the Hawkins sieve (Williams [7]).

2. Proof of the theorem. The proof requires a sharpening of the analysis of [6] whose notation we retain. The starting point is the fact that the process $\left\{\left(X_{n}, Y_{n}\right), n \geqq 1\right\}$ is Markovian with

$$
P\left(X_{n+1}-X_{n}=j \mid F_{n}\right)=Y_{n}^{-1}\left(1-Y_{n}^{-1}\right)^{j-1}, \quad j \geqq 1,
$$

$F_{n}$ being the $\sigma$-field generated by $\left(X_{j}, Y_{j}\right), j \leqq n$.

Set

$$
Z_{n}=X_{n}-1, \quad U_{n+1}=\left(Z_{n+1}-Z_{n}\right) Y_{n}^{-1}, \quad n \geqq 1 .
$$

In [6], Proposition 2, it is shown that if $H_{n}=\log Z_{n}-Y_{n}, n \geqq 1$, then

$$
H_{n}=\log L+O\left(n^{-\frac{1}{2}+\varepsilon}\right) \text { a.s. }
$$

for any $\varepsilon>0$, and we shall strengthen this to show that

$$
H_{n}=\log L+\delta(n)\left(2 n^{-1} \log \log n\right)^{\frac{1}{2}} \quad \text { a.s. , }
$$

where $\lim \sup _{n \rightarrow \infty} \delta(n)=+1$ a.s., $\lim \inf _{n \rightarrow \infty} \delta(n)=-1$ a.s.

Writing $\alpha_{n}=Y_{n} Z_{n}{ }^{-1}, \beta_{n}=Y_{n} Z_{n+1}^{-1}$, we have from [6] that $\alpha_{n}=O\left(n^{-1}\right)$ a.s., $\beta_{n}=O\left(n^{-1}\right)$ a.s. as $n \rightarrow \infty$ and then

where

$$
\begin{aligned}
H_{n+1}-H_{n} & =\log \left(1+\alpha_{n} U_{n+1}\right)-\beta_{n} \\
& =\alpha_{n} U_{n+1}-\beta_{n}+R_{n}
\end{aligned}
$$

$$
\left|R_{n}\right| \leqq \alpha_{n}{ }^{2} U_{n+1}^{2} \quad \text { a.s. }
$$


Further,

so that

$$
\left|\alpha_{n}-\beta_{n}\right|=\left|\alpha_{n} \beta_{n} U_{n+1}\right| \leqq \alpha_{n}{ }^{2}\left|U_{n+1}\right|
$$

where

$$
H_{n+1}-H_{n}=\alpha_{n}\left(U_{n+1}-1\right)+S_{n},
$$

$$
\left|S_{n}\right| \leqq \alpha_{n}^{2}\left(\left|U_{n+1}\right|+U_{n+1}^{2}\right)=O\left(n^{-2} \log n\right) \text { a.s. , }
$$

since, from [6], $\left|U_{n+1}\right|=O(\log n)$ a.s. Then

$$
\begin{aligned}
H_{n+1} & =\sum_{k=1}^{n}\left(H_{k+1}-H_{k}\right)-2 \\
& =\sum_{k=1}^{n} \alpha_{k}\left(U_{k+1}-1\right)+\sum_{k=1}^{n} S_{k}-2
\end{aligned}
$$

and, since $H_{n} \rightarrow_{\text {a.s. }} \log L$ (finite a.s.) as $n \rightarrow \infty$ and $\sum_{1}^{\infty}\left|S_{k}\right|<\infty$ a.s. in view of (2),

$$
H_{n}-\log L=\sum_{k=n}^{\infty} \alpha_{k}\left(U_{k+1}-1\right)+\sum_{k=n}^{\infty} S_{k} .
$$

Now, from (2),

$$
\begin{aligned}
\left|\left(2 n^{-1} \log \log n\right)^{-\frac{1}{2}} \sum_{k=n}^{\infty} S_{k}\right| & =O\left(n^{\frac{1}{2}}(\log \log n)^{-\frac{1}{2}} \sum_{k=n}^{\infty} k^{-2} \log k\right) \\
& =o(1) \text { a.s. }
\end{aligned}
$$

Furthermore,

$$
\sum_{k=n}^{\infty} \alpha_{k}\left(U_{k+1}-1\right)=\sum_{k=n}^{\infty} k^{-1}\left(U_{k+1}-1\right)+\sum_{k=n}^{\infty}\left(\alpha_{k}-k^{-1}\right)\left(U_{k+1}-1\right)
$$

and it is easily seen that

$$
E\left(U_{n+1}-1 \mid F_{n}\right)=0 \text { a.s. , }
$$

so that the $\left\{U_{n+1}-1, n \geqq 1\right\}$ are martingale differences. To prove that

$$
\lim _{n \rightarrow \infty} n^{\frac{1}{2}}(\log \log n)^{-\frac{1}{2}} \sum_{k=n}^{\infty}\left(\alpha_{k}-k^{-1}\right)\left(U_{k+1}-1\right)=0 \quad \text { a.s. , }
$$

it suffices, upon noting that the $\alpha_{n}$ are $F_{n}$-measurable so that the $\left\{\left(\alpha_{k}-k^{-1}\right)\left(U_{k+1}-1\right), k \geqq 1\right\}$ are still martingale differences, to show that (e.g. Lemma 1 of Heyde [4])

$$
\sum_{k=1}^{\infty} k^{\frac{1}{2}}\left(\alpha_{k}-k^{-1}\right)\left(U_{k+1}-1\right) \text { converges a.s. }
$$

This holds if (e.g. Doob [1], page 320)

$$
\sum_{k=1}^{\infty} k\left(\alpha_{k}-k^{-1}\right)^{2} E\left[\left(U_{k+1}-1\right)^{2} \mid F_{k}\right]<\infty \quad \text { a.s. , }
$$

and hence if

$$
\sum_{k=1}^{\infty} k\left(\alpha_{k}-k^{-1}\right)^{2}<\infty \text { a.s. }
$$

since $E\left[\left(U_{k+1}-1\right)^{2} \mid F_{k}\right]=1-Y_{k}^{-1}$ a.s. But,

$$
\alpha_{k}-k^{-1}=Z_{k}^{-1}\left(Y_{k}-k^{-1} X_{k}+k^{-1}\right)=o\left((\log \log k)(k \log k)^{-1}\right) \quad \text { a.s. , }
$$

using the theorem of Heyde [3] and hence (7) and consequently (6) holds. 
We now apply the iterated logarithm result of Theorem 1(b) of Heyde [4] to the tail sum $\sum_{k=n}^{\infty} k^{-1}\left(U_{k+1}-1\right)$ of martingale differences. We have

$$
s_{n}^{2}=\sum_{k=n}^{\infty} k^{-2} E\left[E\left\{\left(U_{k+1}-1\right)^{2} \mid F_{k}\right\}\right]=\sum_{k=n}^{\infty} k^{-2} E\left(1-Y_{k}^{-1}\right) \sim n^{-1} \text { a.s. }
$$

as $n \rightarrow \infty$ and

$$
\sum_{n=1}^{\infty} n^{-1}\left[\left(U_{n+1}-1\right)^{2}-E\left\{\left(U_{n+1}-1\right)^{2} \mid F_{n}\right\}\right] \text { converges a.s. , }
$$

again using [1], page 320 , since $E\left(U_{n+1}-1\right)^{4} \leqq 25$ for all $n \geqq 1$. This last result follows from

$$
\begin{aligned}
E\left(U_{n+1}-1\right)^{4} & =E\left[E\left\{\left(U_{n+1}-1\right)^{4} \mid F_{n}\right\}\right] \\
& =E\left[E\left\{\left(\frac{X_{n+1}-X_{n}}{Y_{n}}-1\right)^{4} \mid F_{n}\right\}\right] \\
& =E\left[\sum_{j=1}^{\infty}\left(j Y_{n}^{-1}-1\right)^{4} Y_{n}^{-1}\left(1-Y_{n}^{-1}\right)^{j-1}\right] \\
& \leqq E\left[\sum_{j=1}^{Y} Y_{n}^{-1}\left(1-Y_{n}^{-1}\right)^{j-1}+\sum_{j=Y_{n}+1}^{\infty}\left(j Y_{n}^{-1}\right)^{4} Y_{n}{ }^{-1}\left(1-Y_{n}{ }^{-1}\right)^{j-1}\right] \\
& \leqq 1+E Y_{n}^{-5} \sum_{j=1}^{\infty} j^{4}\left(1-Y_{n}^{-1}\right)^{j-1} \\
& \leqq 1+24 E Y_{n}^{-5}\left[1-\left(1-Y_{n}^{-1}\right)\right]^{-5} \leqq 25 .
\end{aligned}
$$

The conditions required for the use of Theorem 1(b), Corollary 1 and Corollary 2 of [4] are then simply verified. In particular,

$$
s_{n}^{-2} \sum_{k=n}^{\infty} k^{-2} E\left\{\left(U_{k+1}-1\right)^{2} \mid F_{k}\right\} \rightarrow_{\text {a.s. }} 1,
$$

$E\left[\left|U_{j}-1\right| I\left(j^{-1}\left|U_{j}-1\right|>\varepsilon s_{j}\right)\right] \leqq 25\left(\varepsilon j s_{j}\right)^{-3}$, and

so that

$$
E\left(U_{j}-1\right)^{2} I\left(j^{-1}\left|U_{j}-1\right|>\varepsilon s_{n}\right) \leqq 25\left(\varepsilon j s_{n}\right)^{-2}
$$

$$
\begin{aligned}
& \sum_{j=1}^{\infty} s_{j}^{-1} E\left[j^{-1}\left|U_{j}-1\right| I\left(j^{-1}\left|U_{j}-1\right|>\varepsilon s_{j}\right)\right]<\infty, \quad \text { and } \\
& s_{n}^{-2} \sum_{j=n}^{\infty} E\left[j^{-2}\left(U_{j}-1\right)^{2} I\left(j^{-1}\left|U_{j}-1\right|>\varepsilon s_{n}\right)\right] \rightarrow 0
\end{aligned}
$$

as $n \rightarrow \infty$. We then obtain that the limsup as $n \rightarrow \infty$ of $\left(2 n^{-1} \log \log n\right)^{-\frac{1}{2}} \sum_{k=n}^{\infty} k^{-1}\left(U_{k+1}-1\right)$ is +1 a.s. while the $\lim \inf$ is -1 a.s. The result (1) then follows via (3), (4), (5) and (6).

We now exponentiate (1) and rewrite it in the form

$$
Z_{n}=L e^{Y}\left\{1+\eta(n)\left(2 n^{-1} \log \log n\right)^{\frac{1}{2}}\right\}
$$

where $\lim \sup _{n \rightarrow \infty} \eta(n)=+1$ a.s., $\lim \inf _{n \rightarrow \infty} \eta(n)=-1$ a.s.

Next we have

$$
Z_{n+1} Y_{n+1}^{-1}-Z_{n} Y_{n}^{-1}=U_{n+1}-Y_{n+1}^{-1}, \quad n \geqq 1,
$$

so that summation gives

$$
Z_{n} Y_{n}^{-1}=\sum_{k=2}^{n}\left(U_{k}-1\right)+n-\sum_{k=1}^{n} Y_{k}^{-1}
$$

The martingale law of the iterated logarithm (e.g. Heyde and Scott [5], Corollary 1) applies to $\sum_{k=2}^{n}\left(U_{k}-1\right)$ to give

$$
\begin{aligned}
& \lim \sup _{n \rightarrow \infty}(2 n \log \log n)^{-\frac{1}{2}} \sum_{k=2}^{n}\left(U_{k}-1\right)=+1 \text { a.s., } \\
& \lim \inf _{n \rightarrow \infty}(2 n \log \log n)^{-\frac{1}{2}} \sum_{k=2}^{n}\left(U_{k}-1\right)=-1 \text { a.s., }
\end{aligned}
$$


since

$$
\sum_{k=1}^{n} E\left(U_{k}-1\right)^{2}=\sum_{k=1}^{n}\left(1-E Y_{k}^{-1}\right) \sim n \quad \text { as } \quad n \rightarrow \infty
$$

and $E\left(U_{k}-1\right)^{4} \leqq 25$ while (8) and $E\left[\left(U_{k+1}-1\right)^{2} \mid F_{k}\right]=1-Y_{k}^{-1}$ a.s. ensure that $n^{-1} \sum_{k=1}^{n}\left(U_{k}-1\right)^{2} \rightarrow_{\text {a.s. }} 1$ as $n \rightarrow \infty$. Then, from (10),

$$
\begin{aligned}
& \lim \sup _{n \rightarrow \infty}(2 n \log \log n)^{-\frac{1}{2}}\left(Z_{n} Y_{n}^{-1}-n+\sum_{k=1}^{n} Y_{k}^{-1}\right)=+1 \quad \text { a.s. , } \\
& \lim \inf _{n \rightarrow \infty}(2 n \log \log n)^{-\frac{1}{2}}\left(Z_{n} Y_{n}^{-1}-n+\sum_{k=1}^{n} Y_{k}^{-1}\right)=-1 \quad \text { a.s., }
\end{aligned}
$$

and using (9) and (11) we obtain

$$
\begin{aligned}
\limsup & (2 n \log \log n)^{-\frac{1}{2}}\left|L e^{Y} n Y_{n}^{-1}-n+\sum_{k=1}^{n} Y_{k}{ }^{-1}\right| \\
\leqq & \lim \sup _{n \rightarrow \infty}(2 n \log \log n)^{-\frac{1}{2}}\left|L e^{Y} Y_{n}^{-1}-Z_{n} Y_{n}^{-1}\right| \\
& \quad+\lim \sup _{n \rightarrow \infty}(2 n \log \log n)^{-\frac{1}{2}}\left|Z_{n} Y_{n}^{-1}-n+\sum_{k=1}^{n} Y_{k}{ }^{-1}\right| \\
\leqq & \text { a.s. }
\end{aligned}
$$

The remainder of the proof parallels that of [6]. We extend the random function $Y$ from $\{1,2,3, \cdots\}$ to $(1, \infty)$ by linear interpolation,

$$
Y_{t}=Y_{n}+(t-n)\left(Y_{n+1}-Y_{n}\right), \quad n \leqq t \leqq n+1 .
$$

Then, it is easily checked that

$$
L Y_{t}^{-1} e^{Y}=\int_{1}^{t}\left(1-Y_{s}^{-1}\right) d s+f(t)
$$

where $f(t)=(2 t \log \log (t \vee 3))^{\frac{1}{2}} \alpha(t)$ with $\lim \sup _{t \rightarrow \infty}|\alpha(t)| \leqq 2$ a.s. Furthermore,

$$
\begin{aligned}
{\left[L \operatorname{li}\left(\exp Y_{s}\right)\right]_{1}{ }^{t}=} & \int_{1}^{t}\left(1-Y_{s}^{-1}\right) d\left(L Y_{s}^{-1} e^{Y} s\right) \\
= & t-1+\int_{1}^{t} f^{\prime}(s)\left(1-Y_{s}^{-1}\right)^{-1} d s \\
= & t-1+f(t)\left(1-Y_{t}^{-1}\right)^{-1}-f(1)\left(1-Y_{1}^{-1}\right)^{-1} \\
& \quad-\int_{1}^{t} f(s) Y_{s}^{\prime}\left(Y_{s}-1\right)^{-2} d s
\end{aligned}
$$

while

$$
\left|f(s) Y_{s}^{\prime}\left(Y_{s}-1\right)^{-2}\right|=O\left((\log \log s)^{\frac{1}{2}} s^{-\frac{1}{2}}(\log s)^{-2}\right) \quad \text { as } \quad s \rightarrow \infty
$$

so that

$$
\left(t^{\frac{1}{2}} \log \log t\right)^{-1} \int_{1}^{t}\left|f(s) Y_{s}^{\prime}\left(Y_{s}-1\right)^{-2}\right| d s=o(1) \quad \text { as } \quad t \rightarrow \infty
$$

and hence

$$
\lim \sup _{t \rightarrow \infty}(2 t \log \log t)^{-\frac{1}{2}}\left|L \operatorname{li}\left(e^{Y_{t}}\right)-t\right| \leqq 2 \text { a.s. }
$$

The result of the theorem then follows from (9) and (12) since (9) gives

$$
\lim \sup _{n \rightarrow \infty}(2 n \log \log n)^{-\frac{1}{2}}\left|L \operatorname{li}\left(Z_{n} L^{-1}\right)-L \operatorname{li}\left(e^{Y} n\right)\right| \leqq 1 \text { a.s. }
$$

\section{REFERENCES}

[1] Dooв, J. L. (1953). Stochastic Processes. Wiley, New York.

[2] Hawkins, D. (1958). The random sieve. Math. Mag. 31 1-3.

[3] Heyde, C. C. (1976). On asymptotic behaviour for the Hawkins random sieve. Proc. Amer. Math. Soc. 56 277-280. 
[4] Heyde, C. C. (1977). On central limit and iterated logarithm supplements to the martingale convergence theorem. J. Appl. Probability 14 758-775.

[5] Heyde, C. C. and Scotr, D. J. (1973). Invariance principles for the law of the iterated logarithm for martingales and processes with stationary increments. Ann. Probability $1428-436$.

[6] Neudecker, W. and Williams, D. (1974). The "Riemann hypothesis" for the Hawkins random sieve. Composito Math. 29 197-200.

[7] Williams, D. (1974). A diffusion process motivated by the sieve of Eratosthenes. Bull. London Math. Soc. 6 155-164.

[8] Wunderlich, M. C. (1974). A probabilistic setting for prime number theory. Acta Arith. 26 59-81.

[9] Wunderlich, M. C. (1976). The prime number theorem for random sequences. J. Number Theory 8 369-371.

Division of Mathematics and Statistics CSIRO

P.O. Box 1965, Canberra City

A.C.T. 2601

Australia 